\title{
Vorstandswahlen der Arbeitsgemeinschaften der DRG auf dem 99. Deutschen Röntgenkongress, 9. - 12. Mai 2018 in Leipzig
}

Auf dem diesjährigen Röntgenkongress finden für folgende Arbeitsgemeinschaften Vorstandswahlen statt:

- Deutsche Gesellschaft für Interventionelle Radiologie und minimal-invasive Therapie - DeGIR

- AG Gastrointestinal-/Abdominaldiagnostik

- AG Herz- und Gefäßdiagnostik

- AG Informationstechnologie (AGIT)

- AG Methodik und Forschung (AGMF)

- AG Mammadiagnostik

- AG Physik und Technik (APT)

- AG Thoraxdiagnostik
Entsprechend der Arbeitsordnung der Arbeitsgemeinschaften bedürfen die zur Wahl vorgeschlagenen Kandidaten der Zustimmung des Vorstandes der DRG (nihil obstat). Die Mitglieder der oben genannten Arbeitsgemeinschaften werden hiermit eingeladen, Kandidaten für den Vorstand der Wahlperiode 2018 - 2020 vorzuschlagen. Die Vorschläge müssen schriftlich eingereicht werden und sollten das Einverständnis der vorgeschlagenen Kandidaten enthalten.
Deutsche Gesellschaft für Interventionelle Radiologie und minimal-invasive Therapie (DeGIR)

Der Vorstand der Deutschen Gesellschaft für Interventionelle Radiologie und minimal-invasive Therapie (DeGIR) bittet seine Mitglieder um Kandidatenvorschläge für die Vorstandswahl 2018. Als Kandidaten können nur Mitglieder vorgeschlagen werden, die am 1. März 2018 ordentliche Mitglieder der DeGIR waren. Vorschläge bedürfen der Schriftform und sind bis spätestens 12. März 2018 an den Vorstand der DeGIR 
zu Händen des Vorsitzenden, Prof. Dr. Christian Strosczcynski, Regensburg oder an die Geschäftsstelle der DRG z. Hd. Frau Mirzanli zu senden.

\section{AG Gastrointestinal-/ Abdominaldiagnostik}

Der Vorstand der AG Gastrointestinal-/Abdominaldiagnostik bittet seine Mitglieder um Kandidatenvorschläge für die Vorstandswahl 2018. Als Kandidaten können nur Mitglieder vorgeschlagen werden, die am 1. März 2018 ordentliche Mitglieder der Arbeitsgemeinschaft waren. Vorschläge bedürfen der Schriftform und sind bis spätestens 12. März 2018 an den Vorstand der AG zu Händen des Vorsitzenden, Prof. Dr. Andreas Schreyer, Regensburg oder an die Geschäftsstelle der DRG z. Hd. Herrn Dr. Völker zu senden.

\section{AG Herz- und Gefäßdiagnostik}

Der Vorstand der AG Herz- und Gefäßdiagnostik bittet seine Mitglieder um Kandidatenvorschläge für die Vorstandswahl 2018. Als Kandidaten können nur Mitglieder vorgeschlagen werden, die am 1. März 2018 ordentliche Mitglieder der Arbeitsgemeinschaft waren. Vorschläge bedürfen der Schriftform und sind bis spätestens 12. März 2018 an den Vorstand der Arbeitsgemeinschaft zu Händen des Vorsitzenden, Prof. Dr. Joachim Lotz, Göttingen oder an die Geschäftsstelle der DRG z. Hd. Herrn Dr. Völker zu senden.

\section{AG Informationstechnologie}

Der Vorstand der AG Informationstechnologie bittet seine Mitglieder um Kandidatenvorschläge für die Vorstandswahl 2018. Als Kandidaten können nur Mitglieder vorgeschlagen werden, die am 1. März 2018 ordentliche Mitglieder der Arbeitsgemeinschaft waren. Vorschläge bedürfen der Schriftform und sind bis spätestens 12. März 2018 an den Vorstand der Arbeitsgemeinschaft zu Händen des Vorsitzenden Prof. Dr. Thomas Hackländer, Wuppertal, oder an die Geschäftsstelle der DRG z. Hd. Herrn Dr. Völker zu senden.

\section{AG Methodik und Forschung}

Der Vorstand der AG Methodik und Forschung bittet seine Mitglieder um Kandidatenvorschläge für die Vorstandswahl 2018. Als Kandidaten können nur Mitglieder vorgeschlagen werden, die am 1. März 2018 ordentliche Mitglieder der Arbeitsge- meinschaft waren. Vorschläge bedürfen der Schriftform und sind bis spätestens 12. März 2018 an den Vorstand der Arbeitsgemeinschaft zu Händen des Vorsitzenden Prof. Dr. Clemens Cyran, München oder an die Geschäftsstelle der DRG z. Hd. Herrn Dr. Völker zu senden. 


\section{AG Mammadiagnostik}

Der Vorstand der AG Mammadiagnostik bittet seine Mitglieder um Kandidatenvorschläge für die Vorstandswahl 2018. Als Kandidaten können nur Mitglieder vorgeschlagen werden, die am 1. März 2018 ordentliche Mitglieder der Arbeitsgemeinschaft waren. Vorschläge bedürfen der Schriftform und sind bis spätestens 12 . März 2018 an den Vorstand der Arbeitsgemeinschaft zu Händen des Vorsitzenden Prof. Dr. Markus Müller-Schimpfle, Frankfurt am Main oder an die Geschäftsstelle der DRG z. Hd. Herrn Dr. Völker zu senden.

\section{AG Physik und Technik}

Der Vorstand der AG Physik und Technik bittet seine Mitglieder um Kandidatenvorschläge für die Vorstandswahl 2018. Als Kandidaten können nur Mitglieder vorgeschlagen werden, die am 1. März 2018 ordentliche Mitglieder der Arbeitsgemeinschaft waren. Vorschläge bedürfen der Schriftform und sind bis spätestens 12. März 2018 an den Vorstand der Arbeitsgemeinschaft zu Händen des Vorsitzenden Herrn Dr. Georg Stamm, Göttingen oder an die Geschäftsstelle der DRG z. Hd. Herrn Dr. Gührs zu senden.

\section{AG Thoraxdiagnostik}

Der Vorstand der AG Thoraxdiagnostik bittet seine Mitglieder um Kandidatenvorschläge für die Vorstandswahl 2018. Als Kandidaten können nur Mitglieder vorgeschlagen werden, die am 1. März 2018 ordentliche Mitglieder der Arbeitsgemeinschaft waren. Vorschläge bedürfen der Schriftform und sind bis spätestens 12. März 2018 an den Vorstand der Arbeitsgemeinschaft zu Händen der Vorsitzenden PD Dr. Julia Ley-Zaporozhan, München oder Prof. Dr. Jens Vogel-Claussen, Hannover, oder an die Geschäftsstelle der DRG z. Hd. Frau Engelhardt zu senden. 